\title{
A Mutant Inducible for Galactitol Utilization in Escherichia coli K 12
}

\author{
By C. E. DELIDAKIS, $†$ M. C. JONES-MORTIMER* AND \\ H. L. KORNBERG \\ Department of Biochemistry, University of Cambridge, Tennis Court Road, \\ Cambridge CB2 1QW, U.K.
}

(Received 6 July 1981)

\begin{abstract}
Galactitol-positive strains of Escherichia coli $\mathrm{K} 12$ are inhibited by the galactitol analogues L-fucitol and 2-deoxy-D-galactitol, but not by D-fucitol; Salmonella typhimurium LT2 is not inhibited by these compounds. Most mutants selected as resistant to either toxic compound are unable to utilize galactitol as carbon source, but a relatively rare class is inducible for the Enzyme II of the galactitol:phosphoenolpyruvate phosphotransferase system, the product of which is D-galactitol 6-phosphate. The lesion in one such mutant maps near met $G$ at about $\min 45$ on the $E$. coli genome.
\end{abstract}

\section{INTRODUCTION}

Some, but not all strains, derived from Escherichia coli $\mathrm{K} 12$ will utilize (meso) galactitol as sole carbon source at $30^{\circ} \mathrm{C}$. Galactitol enters cells of $E$. coli by a phosphoenolpyruvate-dependent phosphotransferase mechanism yielding a galactitol phosphate. The galactitol-specific Enzyme II that catalyses this vectorial phosphorylation is specified by the gene gatA, and is expressed constitutively (Lengeler, 1975a, b; Lengeler \& Steinberger, 1978). The gatA gene is $53 \%$ cotransducible with metG (Woodward \& Charles, 1980), the exact location of which (about min 45) is unknown (Bachmann \& Low, 1980).

While screening a number of compounds for their antibacterial activity we observed that L-fucitol (1-deoxy-D-galactitol) and 2-deoxy-D-galactitol inhibited the growth on glycerol minimal medium of galactitol-positive derivatives of $E$. coli K12. Most mutants selected for resistance to either of the toxic compounds were unable to utilize galactitol as carbon source, but some retained their ability to do so. The properties of one such mutant and the genetic location of the lesion are reported here.

\section{METHODS}

The Escherichia coli strains used are listed in Table 1. Salmonella typhimurium LT2 was obtained from Dr D. A. Smith (University of Birmingham). Strains were grown in Oxoid nutrient broth or in the minimal medium of Ashworth \& Kornberg (1966) solidified with $1.5 \%$ (w/v) Oxoid agar no. 1 if required. Bacteriophage P1-mediated transduction was performed according to Lennox (1955). Phosphotransferase assays were done by the method of Kornberg \& Reeves (1972). L-Fucitol, D-fucitol and 2-deoxy-D-galactitol were prepared by reducing L-fucose, D-fucose and 2-deoxy-D-galactose (all from Sigma) with a small excess of sodium borohydride, and were used as their borate esters. Spontaneous resistant mutants were selected by plating $0.1 \mathrm{ml}$ samples of broth-grown cultures on supplemented minimal medium with $20 \mathrm{mM}$-glycerol as carbon source and approx. $5 \mathrm{~mm}$ inhibitor. Cultures were grown at $30^{\circ} \mathrm{C}$ unless otherwise indicated, and cell yields were determined spectrophotometrically at $680 \mathrm{~nm}$.

$\dagger$ Present address: Department of Biochemistry and Molecular Biology, Harvard University, Cambridge, Massachusetts 02138, U.S.A. 
Table 1. Strains of Escherichia coli used

\begin{tabular}{|c|c|c|}
\hline Strain & Genotype* & Origin or reference \\
\hline JM559 & gat-1 fda $^{\text {ts }}$ hisgnd ${ }^{\Delta}$ & Henderson et al. (1977) \\
\hline JM1761 & gat $^{+}$fda $^{\text {ts }}$ hisgnd ${ }^{\Delta}$ & P1.S183-27T $\times$ JM559 \\
\hline JM 1764 & gat-2 fda ${ }^{\text {ts }}$ hisgnd $d^{\Delta}$ & JM1761 (this paper) \\
\hline S 183-27T & gat $^{+}$leu his & Robbins (1975); Robbins \& Rotman (1975) \\
\hline SB 1803 & gat $^{+}$metG thr leu his pro & Blumenthal (1972) \\
\hline W6 & gat-1 met $B$ & Lederberg et al. (1952); Bachmann (1972) \\
\hline
\end{tabular}

* The galactitol-negative mutation of strain W6 and its progeny is indicated by the allele number $I$, and the galactitol-inducible mutation of strain JM1764 is indicated by the allele number 2 .

\title{
Table 2. Galactitol:phosphoenolpyruvate phosphotransferase activity
}

\begin{abstract}
Activity, determined at $30^{\circ} \mathrm{C}$ by the method of Kornberg \& Reeves (1972) in the presence of $4 \mathrm{~mm}$-galactitol, is expressed in $\mathrm{nmol} \mathrm{min}^{-1}$ (mg dry mass) $)^{-1}$. The means, standard deviations and numbers of replicate cultures are given.
\end{abstract}

\begin{tabular}{lclr} 
Strain & Genotype & $\begin{array}{l}\text { Carbon source } \\
\text { for growth }\end{array}$ & \multicolumn{2}{c}{ Activity } \\
JM559 & gat-1 & Galactitol + lactate & $<0.6 \quad(2)$ \\
JM1761 & gat $^{+}$ & Lactate & $4.2 \quad(2)$ \\
JM1761 & gat $^{+}$ & Galactitol & $4.4 \quad(2)$ \\
JM1764 & gat -2 & Lactate & $1.2 \pm 0.6(4)$ \\
JM1764 & gat -2 & Galactitol & $3.6 \pm 0.5(4)$
\end{tabular}

\section{RESULTS}

\section{Isolation and properties of mutants}

Mutants of strain JM1761 $\left(\mathrm{Gat}^{+}\right)$selected on glycerol for resistance to either L-fucitol or 2-deoxy-D-galactitol were always resistant to the other compound. All grew well on glucose at $30{ }^{\circ} \mathrm{C}$ (but not at $40^{\circ} \mathrm{C}$ because of the temperature-sensitive $f d a^{\text {ts }}$ lesion; Böck \& Neidhardt, 1966). The large majority resembled the galactitol Enzyme II-defective strain JM559 in being unable to utilize galactitol as carbon source at $30^{\circ} \mathrm{C}$ and in being resistant to inhibition by galactitol during growth on glycerol at $40^{\circ} \mathrm{C}$. One L-fucitol-resistant mutant, strain JM1764, retained the ability to grow on galactitol $(10 \mathrm{mM})$ as sole carbon source at nearly the same rate as the parental strain (mean doubling times $277 \mathrm{~min}$ and $246 \mathrm{~min}$, respectively).

Salmonella typhimurium LT2 was not inhibited by either of these galactitol analogues, though it grew with galactitol as sole carbon source. D-Fucitol (6-deoxy-D-galactitol) did not inhibit either E. coli or S. typhimurium, nor did any strain tested use it as a carbon source.

\section{Assay of galactitol phosphotransferase activity}

Galactitol phosphotransferase activity was assayed in strains JM559, JM1761 and JM1764 grown on various carbon sources and rendered permeable with toluene, with the results shown in Table 2. Once a rate of NADH oxidation in the presence of $4 \mathrm{~mm}$-galactitol had been determined, glucose $(5 \mathrm{~mm})$ was added to the reaction mixture. This further stimulated the rate of NADH oxidation to $10-20 \mathrm{nmol} \mathrm{min}^{-1}$ (mg dry mass) ${ }^{-1}$. It is evident from Table 2 that the specific activities of the galactitol phosphotransferase systems in the three mutants differed greatly and that JM559 is Gat ${ }^{-}$, JM1764 is Gat ${ }^{+}$but inducible, and JM1761 is $\mathrm{Gat}^{+}$and constitutive.

\section{Genetic location of the lesion in strain JM1764}

Bacteriophage P1 grown on strain JM1764 was used to transduce strain JM559 to Gat ${ }^{+}$. Of 75 transductants examined $9 \%$ were resistant to L-fucitol, as was the donor strain. This 
indicates that the lesion is linked, though not closely, to the gat locus of strain JM559. This gat mutation was mapped by growing bacteriophage P1 on strain JM559 and using it to transduce strain SB1803 to $\mathrm{MetG}^{+}$. Of 80 transductants examined $49 \%$ were $\mathrm{Gat}^{-}$.

\section{DISCUSSION}

Since phosphate esters of sugars and hexitols are toxic to cells that accumulate them (reviewed by Ferenci \& Kornberg, 1973), analogues of such compounds that are modified in such a way as to prevent catabolism are toxic if they can be taken up and phosphorylated (Kornberg \& Smith, 1972; Miles \& Pirt, 1973). If the compound enters by a phosphotransferase mechanism the cell may mutate to acquire resistance only by loss of the phosphotransferase system under the conditions of the experiment. This may be achieved in two different ways: either the cell may lose a structural gene for the system, or it may fail to express that gene. The larger class of $L$-fucitol-resistant mutants, which are indistinguishable in phenotype from strain JM559, are presumably of the former type. Strain JM1764, since it can grow on galactitol as carbon source and can express a galactitol phosphotransferase activity (Table 2), might be a mutant of the latter class.

Like S. typhimurium LT2, strain JM1764 was galactitol-positive and resistant to L-fucitol and 2-deoxy-D-galactitol. Lengeler \& Steinberger (1978) have shown that $S$. typhimurium LT2, unlike $E$. coli $\mathrm{K} 12$, expresses the galactitol phosphotransferase and D-galactitol6-phosphate dehydrogenase inducibly. This suggested that strain JM1764 might also be inducible for the galactitol phosphotransferase. The results in Table 2 show that this is so. That higher phosphotransferase activity was always obtained when glucose was added as substrate subsequent to galactitol indicates that the galactitol Enzyme II, rather than the phospho-carrier protein $\mathrm{HPr}$ or the Enzyme I of the phosphotransferase system, is rate limiting in the assay.

Since L-fucitol and 2-deoxy-D-galactitol are toxic only to strains that express the galactitol phosphotransferase constitutively they cannot be inducers of the phosphotransferase: the inducer thus requires hydroxyl groups at both C-1 and C-2. That D-fucitol (which has no hydroxyl group on C-6 and hence cannot be phosphorylated there) does not inhibit constitutive strains (and is not used as a carbon source) confirms that the product of the phosphotransferase is D-galactitol 6-phosphate rather than D-galactitol 1-phosphate (Lgalactitol 6-phosphate).

Strain W6 (Lederberg et al., 1952) and all of its progeny that we have tested (results not shown) are galactitol-negative, so that the gat-1 mutation in it and its derivative strain JM559 is of some antiquity. The lesion may have arisen by the unintentional selection of faster-growing colonies on partially hydrolysed agar medium above the permissive temperature for growth on galactitol. That the lesion we have isolated in strain JM1764 is located close to the genes gatA and gatD for the galactitol phosphotransferase and galactitol-6-phosphate dehydrogenase was predicted from what is known of the organization of the very similar operons for the catabolism of mannitol and sorbitol (Lengeler, 1975 a).

We have not sought to establish the reason for the constitutive expression of the galactitol operon in wild-type cells. The confusing mapping data in this region of the genome (Bachmann \& Low, 1980) has deterred any more extensive mapping of the lesion. The observation that $E$. coli $\mathrm{K} 12$ strains will mutate to express the galactitol operon inducibly suggests that the prototype strain $\mathrm{K} 12$ should be regarded as a constitutive mutant of some ancestral organism.

We are grateful to Drs B. J. Bachmann and P. J. F. Henderson for providing strains, to Mr B. E. Harrington for isolating the inducible mutant JM1764, and to $\mathrm{Dr}$ J. A. Sokatch for gifts of D- and L-fucose and 2-deoxy-D-galactose. This work was supported by the Science Research Council under grant GR/A/22482. 


\section{REFERENCES}

Ashworth, J. M. \& Kornberg, H. L. (1966). The anaplerotic fixation of carbon dioxide by Escherichia coli. Proceedings of the Royal Society B165, 179-188.

BachmanN, B. J. (1972). Pedigrees of some mutant strains of Escherichia coli K12. Bacteriological Reviews 36, 525-557.

BachmanN, B. J. \& Low, K. B. (1980). Linkage map of Escherichia coli $\mathrm{K} 12$, edition 6. Microbiological Reviews 44, 1-56.

BLUMENTHAL, T. (1972). P1 transduction: formation of heterogenotes upon cotransduction of bacterial genes with a P2 prophage. Virology 47, 76-93.

Böck, A. \& NeIDHARDT, F. C. (1966). Isolation of a mutant of Escherichia coli with a temperaturesensitive fructose 1:6-diphosphate aldolase activity. Journal of Bacteriology 92, 470-476.

Ferenci, T. \& KornberG, H. L. (1973). The utilisation of fructose by Escherichia coli. Properties of a mutant defective in fructose 1-phosphate kinase activity. Biochemical Journal 132, 341-347.

Henderson, P. J. F., Giddens, R. A. \& JonesMORTIMER, M. C. (1977). Transport of galactose, glucose and their molecular analogues by Escherichia coli K12. BLLD supplementary publication SUP 50074 to Biochemical Journal 162 , 309-320.

Kornberg, H. L. \& Reeves, R. E. (1972). Correlation between hexose transport and phosphotransferase activity in Escherichia coli. Biochemical Journal 126, 1241-1243.

KoRNBERG, H. L. \& SMITH, J. (1972). Genetic control of glucose uptake by Escherichia coli. FEBS Letters 20, 270-272.

Lederberg, J., Cavalli, L. L. \& Lederberg, E. M.
(1952). Sex compatibility in Escherichia coli. Genetics 37, 720-730.

LENGELER, J. (1975a). Mutations affecting transport of the hexitols D-mannitol, D-glucitol and galactitol in Escherichia coli: isolation and mapping. Journal of Bacteriology 124, 26-38.

LENGELER, J. (1975b). Nature and properties of hexitol transport systems in Escherichia coli. Journal of Bacteriology 124, 39-47.

Lengeler, J. \& Steinberger, H. (1978). Analysis of the regulatory mechanisms controlling the synthesis of the hexitol transport systems in Escherichia coli K12. Molecular and General Genetics 164, 163169.

LENNOX, E. S. (1955). Transduction of linked genetic characters of the host by bacteriophage P1. Virology 1, 190-206.

Miles, R. J. \& PIRT, S. J. (1973). Inhibition by 3-deoxy-3-fluoro-D-glucose of the utilization of lactose and other carbon sources by Escherichia coli. Journal of General Microbiology 76, 305-318.

Robiins, A. R. (1975). Regulation of the Escherichia coli methylgalactoside transport system by gene mglD. Journal of Bacteriology 123, 69-74.

Robilns, A. R. \& RotMAN, B. (1975). Evidence for binding protein-independent substrate translocation by the methylgalactoside transport system of Escherichia coli K12. Proceedings of the National Academy of Sciences of the United States of America 72, 423-427.

Woodward, M. J. \& Charles, H. P. (1980). Genes for ribitol and arabitol utilization, and genes for galactitol utilisation, behave as chromosomal alternatives. Society for General Microbiology Quarterly 7,82 . 